\title{
An Separation of Different Facial Expression Recognition System
}

\author{
Nithin S, Aravindan M B, Abhilash Hegde, Gururaj H L
}

\begin{abstract}
Face recognition is one of the hot topics in the current world and one of the popular topics of computer studies. Today face recognition in the network society and access to digital data is gaining more attention. The facial recognition system technology is a biometric assessment of a human's face. There are many facial recognition techniques that are intended depending on facial expressions extraction, one of which is $3 D$ facial recognition, as well as their fusion,is difficult. During preprocessing measures for picture recognition to remove only expression-specific characteristics from the face and prevent their issues with a convolution neural network. We can also use some theorems such as LBP and Taylor's theorem to model face recognition. In particular, for cloud robots, we can also use this facial recognition on robots. The robot can perform functions and share data between servers and devices. Seven fundamental expressions are used to identify and classify: happiness, shock, fear, disgust, sadness, rage, and a neutral condition. Until now, the recognition rate is quite up to the expectation stage, but it still tries to enhance. To enhance the recognition frequency of facial image recognition, feelings are chosen by the vibrant Bayesian network technique to depict the development of facial awareness in addition to various emotional operations of facial expressions. The ICCA techniques involve various multivariate sets of distinct facial features that could be eyes, nose, and mouth.
\end{abstract}

Index Terms- Facial expression, face recognition, Expression, Emotions.

\section{INTRODUCTION}

Machine learning is the study of algorithms and models used by the system to improve their performance on specific tasks; facial recognition system is a technology capable of identifying or verifying a person or living being from a digital image or a video frame from a video source. Face recognition is one of today's hot topics in both machine learning and research. Face recognition and expression of facial speech in many fields perform a broad role. For face recognition, many algorithms are applied. It is used when issuing identification documents and is most commonly combined with other biometric techniques such as fingerprints.

As robotics range continues to expand, it has become a challenge to write robot software. The fundamental necessity is frequently the same for all robots. Implementing the same multi-robots features allows for unnecessary costs. A robot's face recognition job is a feature to enhance people's robot communication. It comprises of image processing, removal of features, algorithm application and

Revised Manuscript Received on April 12, 2019.

Nithin S,Department of Computer Science and Engineering, Vidyavardhaka College of Engineering, Mysuru, Karnataka, INDIA. (Email: nitinparam356@gmail.com)

Aravindan M B, Department of Computer Science and Engineering, Vidyavardhaka College of Engineering, Mysuru, INDIA.

AbhilashHegde, Department of Computer Science and Engineering, Vidyavardhaka College of Engineering, Mysuru, KarnatakaINDIA.

Gururaj H L, Department of Computer Science and Engineering, Vidyavardhaka College of Engineering, Mysuru, KarnatakaINDIA. lastly system matching. For its storage and identity database, the $3 \mathrm{~d}$ image test is first depicted as a $2 \mathrm{~d}$ depth chart, for speech and forecast facial components from both texture and depth maps are recorded.

In considering distinct topics, these differences are emphasized. Facial expression is a noticeable manifestation of an individual's effective condition, mental activity, intent, character, and psychopathology. FACS (Facial action coding system) is the purpose and inclusive for AU (Action Unit) coding system.

Because of its broad actual world apps, such as CCTV cameras, individual monitoring, and access control, face recognition has increased intensively and more interest in model recognition. Usually, however, the device's pictures are of poor performance. Various human facial expressions, gestures, styles and circumstances of illumination influence the performance of facial images, triggering mistakes in occlusion, translation, and scale, etc. One of the primary difficulties in the assignment of recognizing the high dimensional image room or on less than a big quantity of test data. Many techniques of recognition are being suggested today and some of these techniques of recognition have achieved a high identification frequency and have been successful over the previous few years, but solid techniques of image recognition are still needed with greater identification performance. Maintaining performance and time efficiency in face recognition is so crucial, so it is still in hot study subject lists and there are distinct algorithms and distinct methods to achieve excellent time-complex and efficiency recognition rates.

\section{RELATED WORK}

In [1] the authors perform the intrafacial canonical correlation analysis for image identification using techniques such as ICCA and CCA relying on parameters that various sets of distinct facial characteristics that could be eyes, nose, mouth and their outcomes in that the suggested strategy achieves stronger identification rates than the traditional statistical analysis on the AR face database and the advantage of this approach or research is that it achieves a better recognition rate than previous approaches.

In [2] the authors proposed that Facial Expression Identification System with a linear range of facial corners using techniques such as SVM Canny edge detection Euclidean distance map centered on facial parameters, facial features, Euclidean distance, and seven distinct facial forms and its outcomes in seven facial forms and the benefit of the suggested method. 
Thuthi [3] evaluated that Recognition of facial expression using techniques of action unit classification using methods such as dynamic Bayesian network Gabor wavelet \& SUSAN operator accepted on the basis of parameters that facial forms, different facial expressions and their outcomes in that the suggested strategy is that significant enhancement in facial expressions \& AU identification and the benefit of this approach or research is that it increases the rate of recognition of facial expression \& AU recognition.

Jan et.al [4] suggested that an accurate location of facial components and profound teaching for identification of $3 \mathrm{D}$ facial expression using techniques. In this paper a new scheme for $3 \mathrm{D}$ facial recognition is intended based on precise facial components removal as well as their combination are difficult duties depending on parameters of texture, facial parts 3 dimensions and its outcomes in that the suggested method image 3D facial recognition offers an efficient identification of facial parts and textures and the benefit of this strategy or study that accurate recognition of facial parts can be done.

Chen et.al [5] approach the Convolution Neural network for automatic identification of facial expression using techniques. When recognizing image preprocessing measures to extract only particular phrases from the face and prevent their issues with parameter-based convolution neural network. Recognition of facial expression, neural network and its outcomes in the suggested face expression strategy by using a convolution neural network offers greater precision with large information. This CNN makes recognition at a quick pace and very rapidly detects facial components and enables identification of facial expression in the actual moment with a conventional system.

Ding et.al [6] approaches the recognition of facial expression from image series based on the use of techniques by LBP and Taylor expansion. The LBP and Taylor extension filters are used to identify facial monuments and data sets are used to recognize facial expression based on the model of Local Binary, Taylor's Theorem and its outcomes in that the strategy suggested. Every emotion is recognized, such as anger and fear, and its pace can be produced. To acquire efficient facial recognition and identify expressions depending on their facial tissues whether the individual is in anger or fear and other features using this development of Taylor and LBP.

Sadeghi et.al [7] strategy that face recognition using geometric normalization and depiction of personality using techniques such as set linear template \& facial image normalization. Based on parameters that recognition rate, number of faces identified and outcomes in the suggested method. Experiment outcome demonstrates that designs with a big mouth and eye size are more effective while tiny mouth and eye size are relatively less precise.The primary benefit of this is that it demonstrates which geometric forms are best suited for face identification.
The facial action coding scheme used [8] to monitor and recognize and analyze emotional facial behaviors depending on parameters Threshold values, RGB frequency. Robots are prepared to recognize different peoples' facial feelings in real time. The robot is used for face identification detection and can be used for safety reasons.

Pukhrambam et.al [9] strategy is that facial identification by extracting facial elements in static images. They used machine classifier here to rank images that belong to which class category by comparing trained image vectors depending on parameters that measure Hue Saturation, False Positive and False Negative. Compared to other emotional states, the suggested strategy demonstrates more accuracy for joy and the advantage is that it can remove facial elements and other emotional situations.

\section{RESULTS \& DISCUSSIONS}

Chou and Yang [1] approaches face recognition not only addresses insufficient images of the image strategies issue in practice, such as the PCA, the LDA, and the LRC. This paper contributes to the addition of significant facial features to shape the observation matrix after the canonical relationship between them has been calculated. In order to assess the efficacy of the suggested ICCA facial identification system, studies were performed on the AR face database containing 3510 mug images of 135 topics (76 men and 59 women) with distinct facial gestures, variations in color and partial occlusions, including neutral expression, smile, anger, screaming, screaming, different lighting changes, and two realistic partial occlusions with lighting changes. they cropped face images into four parts manually including original face, eyes, nose, and mouth. and thus the results show that the proposed approach can boost the recognition accuracy more than contemporary popular approach over 3\% under facial expression as shown in figure 1. According to them, the proposed ICCA approach is well-suited for personal identification.

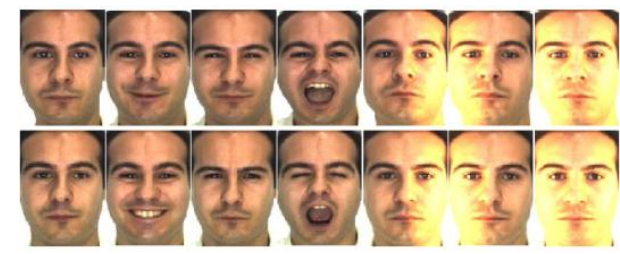

Fig 1. Different facial expressions

Kherchaoui and Houacine [2] used JAFFE (Japanese Female Facial Expression) system training and evaluation database containing 213 facial images with seven facial expressions; six fundamental expressions plus one neutral. Facial expression rates are properly identified at $93.24 \%$ and the highest rate is for the positive and happy expression that achieves $100 \%$ precision.So it's easier to compare the prior paper. The table 1 below demonstrates the outcomes of acceptance in the second document. 
Table 1. Face expression recognition

\begin{tabular}{|l|l|l|l|l|l|l|l|}
\hline State & Anger & Disgr & Fear & Happy & Neutral & Sad & Surprise \\
\hline $\begin{array}{l}\text { Rate } \\
\%\end{array}$ & 90 & 88.88 & 91.66 & 100 & 91.66 & 100 & 90 \\
\hline
\end{tabular}

Thuthi [3] also uses the Jaffe database to calculate and assess the scheme for acceptance of facial expressions. The database includes a few people's expression in it. Every individual has distinct expressions and feelings like astonishment, neutrality, anger, fear, sorrow, disgust, happiness. Then the performance comparison is made with the existing method and the proposed model as shown in figure 2 below and the results of the experiments show that the proposed model achieves substantial improvement in $\mathrm{AU}$ recognition than the existing model in that period.

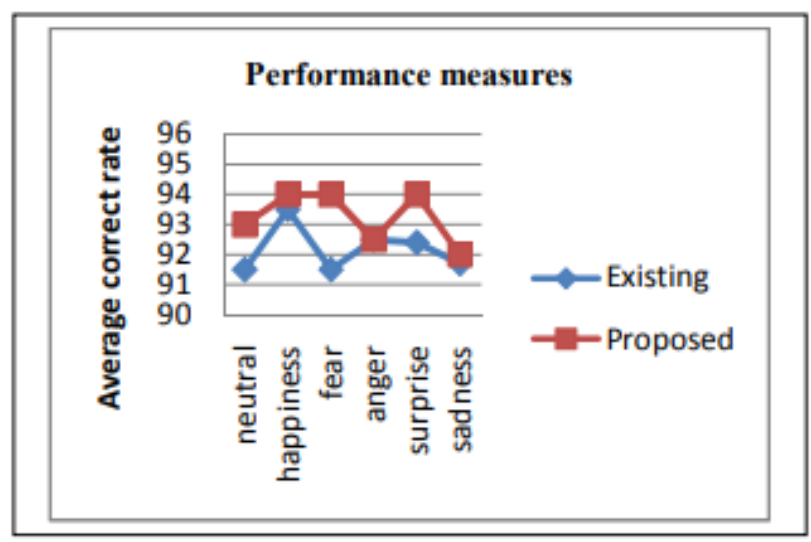

Fig 2. Performance measures of di fferent facial expressions

In [5] here they used to extend $\mathrm{CK}+$ database which contains some video sequences in which each sequence comprises of about 10 to 30 frames and seven types of expressions categories: angry, disgust, neutral, sad, fear, surprise, happy. Each sequence begins with the neutral emotion and then the feelings of the image change through the respective sequence marks. The selected pictures are human faces with evident facial features to acknowledge facial expression. All these Cohn-Kanaded database sequences are used to evaluate the suggested system. In order to check the generalization of the suggested technique, cross-database studies were also carried out. JAFFE database, consisting of distinct images from a few Japanese woman subjects, was also used in tests s was the case in the earlier article. Here, stochastic gradient descent methods are frequently used for model learning and these tests lead to important improvements in awareness compared to others.

In [6] they compare the efficiency of other CK+ dataset research or work with the technique suggested as the prior article also used $\mathrm{CK}+$ storage. Other works or research in their techniques passed through the distinct exam systems. Here they find acceptance of 7-class expression to include the Contempt expression. These experimental findings are compared with other works shown in table 2 below.
Table 2. Comparison of findings with different facial expression

\begin{tabular}{|c|c|c|c|}
\hline Method & $\begin{array}{c}\text { Class } \\
\text { Num. }\end{array}$ & $\begin{array}{c}\text { Accuracy } \\
\text { (\%) }\end{array}$ & Cross valid \\
\hline This work & $\mathbf{6}$ & $\mathbf{9 6 . 5 3}$ & leave-one-subject-out \\
This work & 7 & $\mathbf{9 5 . 0 7}$ & leave-one-subject-out \\
This work & $\mathbf{6}$ & $\mathbf{9 4 . 4 8}$ & 10-fold \\
This work & $\mathbf{6}$ & $\mathbf{9 4 . 1 6}$ & 5-fold \\
Ptucha [14] & 7 & 91.4 & leave-one-subject-out \\
Huang [12] & 7 & 93 & leave-one-subject-out \\
S. Islam [11] & 7 & 90.1 & 10-fold \\
Sanin [13] & 7 & 93.91 & 5-fold \\
Kanade [16] & 7 & 88.33 & leave-one-subject-out \\
\hline
\end{tabular}

In this table, in relation to six fundamental emotions for tests, 6-class techniques with six universal emotions and on including Contempt expression7-class techniques are used. We have come to understand from this table that the highest efficiency is provided by the strategy suggested in this article to compare with current techniques.

\section{Fig 3. Facial geometric model [7]}

Here, they approach for on using geometric variability elimination and Local Binary Patterns facial expression recognition as image texture descriptor [7].

In [8], they created facial analyzers of two NN-based upper and lower operation analyzers, where the upper analyzer analyzes the upper portion of the images and the lower analyzer analyzes the reduced portion of the feelings of the subjects, and these two analyzers reached a strong identification level on the trials facing. Then, when using SVM verbal image recognizer upper AUs and reduced AUs, facial recognizers are inputted into SVM and feelings are detected.

They used 100 face images as the training set in [9] and 60 face images as test material. They consist of various emotions in which these images are trained by SVM classifier in that instruction set images. Now, each images emotional state in the test collection is contrasted and recognized with images in the training set. The table below demonstrates that different facial gestures identification frequency as shown in above table 3 this paper give's result of that significant improvement emotion recognition. 
Table 3 Recognition rate of various facial expressions.

\begin{tabular}{|c|c|c|}
\hline Emotional state & $\begin{array}{c}\text { No. of images } \\
\text { in training set }\end{array}$ & $\begin{array}{c}\text { Recognition } \\
\text { rate of the } \\
\text { images in } \\
\text { testing set }\end{array}$ \\
\hline HAPPY & 30 & $95 \%$ \\
\hline SAD & 10 & $85 \%$ \\
\hline ANGER & 15 & $60 \%$ \\
\hline NEUTRAL & 20 & $75 \%$ \\
\hline DISGUST & 25 & $65 \%$ \\
\hline
\end{tabular}

\section{CONCLUSION}

In recent years, face recognition technology has come a long way, today computers can automatically check recognition data for safe data exchange for monitoring and safety duties and for gaining control. Using the suggested technique, we enhance the image recognition scheme under illumination variety and non-frontal perspective, and it is very easy in terms of calculation, enhanced recognition velocity, without the need for complex scanning, precision dependence is accomplished in this article. So we found that we can attain more precision and velocity of detection on the basis of the above techniques

\section{ACKNOWLEDGEMENTS}

The manuscript is prepared by taking assistance from Accendere Knowledge Management Services Pvt. Ltd, we are thankful to them. We also express our gratitude to our teachers and mentors for guiding us throughout the work.

\section{REFERENCES}

1. Yang-Ting Chou and J.-F. Yang, "Intra-facial-feature canonical correlation analysis for face recognition," in TENCON 2015 - 2015 IEEE Region 10 Conference, 2015, pp. 1-4.

2. S. Kherchaoui and A. Houacine, "Facial expression identification system with Euclidean distance of facial edges," in 2014 6th International Conference of Soft Computing and Pattern Recognition (SoCPaR), 2014, pp. 6-10.

3. D. Thuthi, "Recognition of facial expression using action unit classification technique," in 2014 International Conference on Recent Trends in Information Technology, 2014, pp. 1-5.

4. A. Jan, H. Ding, H. Meng, L. Chen, and H. Li, “Accurate Facial Parts Localization and Deep Learning for 3D Facial Expression Recognition," in 2018 13th IEEE International Conference on Automatic Face \& Gesture Recognition, 2018, pp. 466-472.

5. X. Chen, X. Yang, M. Wang, and J. Zou, "Convolution neural network for automatic facial expression recognition," in 2017 International Conference on Applied System Innovation (ICASI), 2017, pp. 814-817.

6. Y. Ding, Q. Zhao, B. Li, and X. Yuan, "Facial Expression Recognition from Image Sequence Based on LBP and Taylor Expansion," IEEE Access, vol. 5, pp. 19409-19419, 2017.

7. H. Sadeghi, A.-A. Raie, and M.-R. Mohammadi, "Facial expression recognition using geometric normalization and appearance representation," in 2013 8th Iranian Conference on Machine Vision and Image Processing (MVIP), 2013, pp. 159-163.
8. L. Zhang, A. Hossain, and M. Jiang, "Intelligent Facial Action and emotion recognition for humanoid robots," in 2014 International Joint Conference on Neural Networks (IJCNN), 2014, pp. 739-746.

9. M. Pukhrambam, A. Das, and A. Saha, "Facial components extraction and expression recognition in static images," in 2015 International Conference on Green Computing and Internet of Things (ICGCIoT), 2015, pp. 726-731. 